\title{
Study of muon-induced background in MMC detector arrays for the ECHo experiment
}

\author{
A. Göggelmann ${ }^{1, \mathrm{a}}{ }_{\mathbb{O}}$, J. Jochum $^{1}$, L. Gastaldo ${ }^{2}$, C. Velte $^{2}$, F. Mantegazzini ${ }^{2}$ \\ ${ }^{1}$ Physics Institute, University of Tübingen, Tübingen, Germany \\ ${ }^{2}$ Kirchhoff Institute for Physics, Heidelberg University, Heidelberg, Germany
}

Received: 26 January 2021 / Accepted: 16 April 2021 / Published online: 27 April 2021

(C) The Author(s) 2021

\begin{abstract}
For above ground particle physics experiments, cosmic muons are common source of background, not only for direct detector hits, but also for secondary radiation created in neighboring materials. The ECHo experiment has been designed for the determination of the effective electron neutrino mass by the analysis of the endpoint region of the ${ }^{163} \mathrm{Ho}$ electron capture spectrum. The fraction of events occurring in the region of interest of $10 \mathrm{eV}$ below the $Q_{\mathrm{EC}}$ value of about $2.8 \mathrm{keV}$ is only of the order of $10^{-9}$. This means that the background in that region need to be studied, characterized and methods to suppress it need to be developed. We expect a major background contribution to be due to cosmic muons and radiation produced by muons traveling through material around the detectors. To determine the muon-related background in metallic magnetic calorimeters (MMCs) used in the ECHo experiment, we have performed an experiment in which a muon veto was installed around the cryostat used for the operation of the detectors. We analysed the acquired events to investigate the pulse shape of MMC events in coincidence with the muon veto and the rate of multiple coincidences among detector array pixels. With different methods used for identification of muon related events, we studied events generated by muons and secondary radiation depositing energy in the substrate close to the ECHo pixels. In addition, energy depositions of muons and secondary radiation in the detectors was studied via Monte Carlo simulation. At the present status of investigation, we conclude that muon related events will be a negligible background in the region of interest of the ${ }^{163} \mathrm{Ho}$ spectrum.
\end{abstract}

\footnotetext{
a e-mail: alexander.goeggelmann@uni-tuebingen.de (corresponding author)
}

\section{Introduction}

With an estimated flux of 180 muons s$~^{-1} \mathrm{~m}^{-2}$ at sea level [1], cosmogenic muons are a common background issue in a variety of rare events experiments, e.g. experiments for the investigation of neutrino properties [2-5] or experiments searching for dark matter [6,7]. While these experiments are often located underground to reduce the muon flux, the only available method to reduce muon induced events in above ground experiments is the use of a muon veto. Not only direct muon interactions with the detectors can be critical for the experiment, but also the muon induced secondary particles created as products of the interaction of the muons with the setup of the experiment.

The ECHo experiment [8] is an above ground experiment, which measures the full ${ }^{163} \mathrm{Ho}$ electron capture (EC) spectrum to determine the effective electron neutrino mass by using cryogenic metallic magnetic calorimeters (MMCs) $[9,10]$.

The endpoint region of the ${ }^{163} \mathrm{Ho}$ EC spectrum below the $Q_{E C}$ value, which is the maximum energy available for the EC decay, is most effected by the finite neutrino mass [11] [12] (illustrated in Fig. 1). The $Q_{\mathrm{EC}}$ value is given by the mass difference between the ${ }^{163} \mathrm{Ho}$ atom and its daughter atom ${ }^{163} \mathrm{Dy}$. A measurement of $Q_{\mathrm{EC}}$ independent from the EC of ${ }^{163}$ Ho has been obtained using the double Penning Trap SHIPTRAP [13] $Q_{\mathrm{EC}}=\left(2833 \pm 30_{\text {stat }} \pm 15_{\text {sys }}\right) \mathrm{eV}$. In the ECHo- $1 \mathrm{k}$ detector design, the ${ }^{163} \mathrm{Ho}$ source was surrounded by at least $5 \mu \mathrm{m}$ of gold, which ensures a quantum efficiency larger than $99.999 \%$ for the full energy range of the ${ }^{163} \mathrm{Ho}$ spectrum. In the last $10 \mathrm{eV}$ below $Q_{\mathrm{EC}}$, the region of interest (ROI) in this work, only a tiny fraction of all events of the order of $10^{-9}$ are expected (compare to Fig. 1).

In the ECHo-1k phase, 64 on MMC [10] based detector pixels will be used. The ${ }^{163} \mathrm{Ho}$ activity per pixel for the ECHo-1k arrays is about $1 \mathrm{~Bq}$. This low activity was due 
to difficulties in the ${ }^{163} \mathrm{Ho}$ implantation process [14]. Solutions for increasing the activity per pixel have been found and for the second stage of the ECHo experiment, ECHo$100 \mathrm{k}$, an ${ }^{163} \mathrm{Ho}$ activity of $10 \mathrm{~Bq}$ is foreseen. In this phase a count rate of approximately $8 \times 10^{-4}$ count day $^{-1}$ pixel $^{-1}$ in the ROI is expected. We discuss the muon related background in the ROI in relation to an intrinsic pile-up background corresponding to a ${ }^{163} \mathrm{Ho}$ activity per pixel of $10 \mathrm{~Bq}$.

Due to the finite time resolution $\tau_{r}(\cong$ signal rise time) of MMCs of about $300 \mathrm{~ns}$, unresolved pile-up events represents an intrinsic background for this measurement technique, whose rate can be determined with $r_{p u}=A^{2} \cdot \tau_{r}$, with the ${ }^{163}$ Ho activity per detector pixel $A$. Figure 1 shows together with the ${ }^{163} \mathrm{Ho}$ spectrum also the contribution due to the unresolved pile-up events for an expected unresolved pile-up rate of about $2 \times 10^{-4}$ counts day ${ }^{-1}$ pixel $^{-1}$ in the ROI.

The ECHo collaboration aims to reduce all external background source as muon related events or events due to natural radioactivity, so that the dominant background in the endpoint region is represented by unresolved pile-up events. Different approaches to reduce the backgrounds are in development. For this, Monte Carlo simulations are performed to study the influence of muons and radioactivity in the ECHo set-up. In addition, methods for pulse shape analysis are developed to recognize events caused by muons and radioactive decays. Screening measurements are performed and an active muon veto is operated.

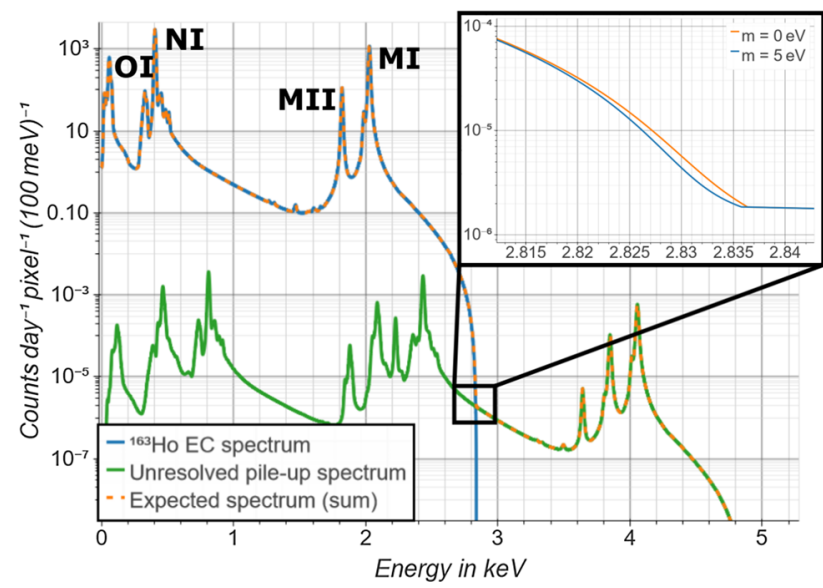

Fig. 1 Theoretical EC spectrum of ${ }^{163} \mathrm{Ho}$ according to the model developed in [11] (blue solid line) with $10 \mathrm{~Bq}$ of ${ }^{163} \mathrm{Ho}$ per pixel. The green solid line represents the unresolved pile-up spectrum calculated as the autoconvolution of the ${ }^{163} \mathrm{Ho}$ spectrum for an unresolved pile-up fraction of $3 \times 10^{-6}$. Insert: Magnification of the end point region of the ${ }^{163} \mathrm{Ho}$ EC spectrum for a vanishing neutrino mass (orange) and a neutrino mass of $5 \mathrm{eV}$ (blue)

\section{Cosmic muons}

The detectors used in the ECHo experiment are metallic magnetic calorimeters which are operated at $\mathrm{mK}$ temperatures in a dilution refrigerator $[9,10]$. The detector arrays used for the discussed simulations and measurements is of the type ECHo-1k [15]. A sketch of this chip is showed in Fig. 2. It consists of a silicon substrate of dimension $10 \mathrm{~mm} \times 5 \mathrm{~mm}$ and thickness $350 \mu \mathrm{m}$ with 36 MMCs with double meander geometry, resulting in 72 pixels positioned in four lines each with 18 pixels. Each pixel consists of a gold absorber of dimension $180 \mu \mathrm{m} \times 180 \mu \mathrm{m} \times 10 \mu \mathrm{m}$, which is on top of a sensor made of $\mathbf{A g}$ :Er of dimension $180 \mu \mathrm{m} \times 180 \mu \mathrm{m} \times 1.35 \mu \mathrm{m}$. In addition, relatively large gold structures, which are important for the thermalization of the chip, are present. Four SQUIDs chips, also microfabricated on a silicon substrate with dimensions of about $6 \mathrm{~mm} \times 2.5 \mathrm{~mm} \times 350 \mu \mathrm{m}$ are positioned very close to the ECHo-1k chip. The electrical connections between the SQUID chips and the detector chip are realized via $\mathrm{Al}$ bonding wires. The five silicon chips are glued to a OFHC copper holder. The copper holder has a thickness of $0.7 \mathrm{~cm}$. The circuit board (in the simulation made of a bisphenol-based epoxy resins) covers the full surface of the copper holder minus the parts where the chips are positioned. The set-up has a T-shape to allow the placement of a magnetic shield, an aluminum tube, which is superconducting at the operation temperature of the detector. The $\mathrm{Al}$ shield has a rectangular aperture cross section of about $2.6 \mathrm{~cm}$ (width) $\times 0.9 \mathrm{~cm}$ (height) with a length of $15 \mathrm{~cm}$ and a wall thickness of about $3 \mathrm{~mm}$ for the top and bottom

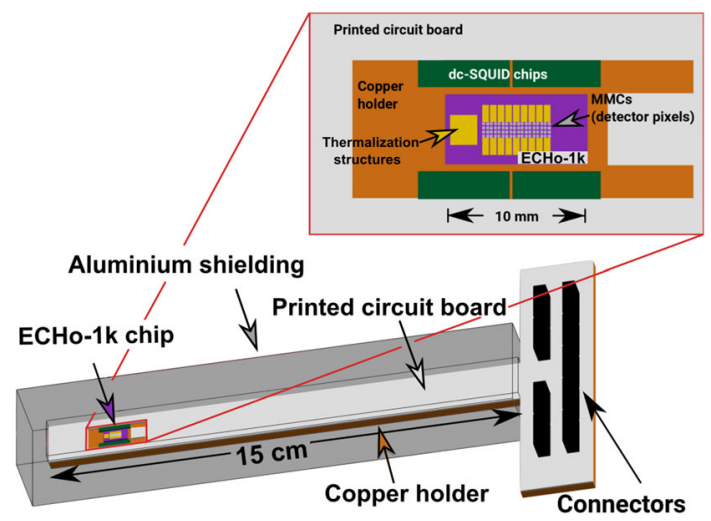

Fig. 2 Simplified design of the ECHo-1k experiment setup used in the simulation. Connectors are placed at a distance of about $15 \mathrm{~cm}$ from the ECHo-1k chip and an aluminum box surrounds the copper holder. On top of the copper holder, the white circuit board, the silicon substrate, with the ECHo detector pixels on top, and the dc-SQUID chips are placed. Insert: Magnification of the part close to the ECHo-1k chip. The purple colored silicon ECHo-1k chip is surrounded by four dcSQUID chips (green). All chips are glued directly on the copper holder. On the ECHo-1k chip the large gold thermalization structured can be seen as well as the four rows of the MMCs (silver colored) 
sides and about $10.3 \mathrm{~mm}$ for the other sides. About $15 \mathrm{~cm}$ away from the detector chip, nine connectors (in the simulation made of carbon with a density of $1 \mathrm{~g} \mathrm{~mol}^{-1}$ ) are mounted.

\subsection{Expected energy deposition of muons passing MMC pixels}

The rate of cosmic muons at the sea level can be quantified to about 180 muons $\mathrm{s}^{-1} \mathrm{~m}^{-2}$. A back-of-the-envelope calculation tells that we would expect 0.5 direct muon hits per day per pixel for absorber areas of $180 \mu \mathrm{m} \times 180 \mu \mathrm{m}$. It is interesting to look at the distribution of the energy deposited by a muon passing through a gold layer for different layer thicknesses. The energy and angular distribution of the arriving muons are given by $[16,17]$. Also, only the energy loss straggling by ionization [18] is considered. Figure 3 shows this energy distribution in the case for MMC absorbers having a thickness of 5, 10, 15 and $20 \mu \mathrm{m}$. Due to the different angles at which the muons are approaching the surface of a MMC pixel, different path lengths occur, ranging from sub $\mu \mathrm{m}$ for muons traveling through an edge to about $260 \mu \mathrm{m}$ for a muon moving along the diagonal. According to Fig. 3, only short muon path lengths in the gold absorber would lead to energy depositions in the region of interest close to the ${ }^{163} \mathrm{Ho}$ EC spectrum end-point of $\sim 2.8 \mathrm{keV}$. Events with energies larger than $10 \mathrm{keV}$ are typically not acquired during the ${ }^{163} \mathrm{Ho}$ spectrum measurement. It has to be noted, that energy loss due to resonances with the atomic shell levels of gold, which are less than $4 \mathrm{keV}$ for the $\mathrm{M}, \mathrm{N}$ and $\mathrm{O}$ shells, are not included

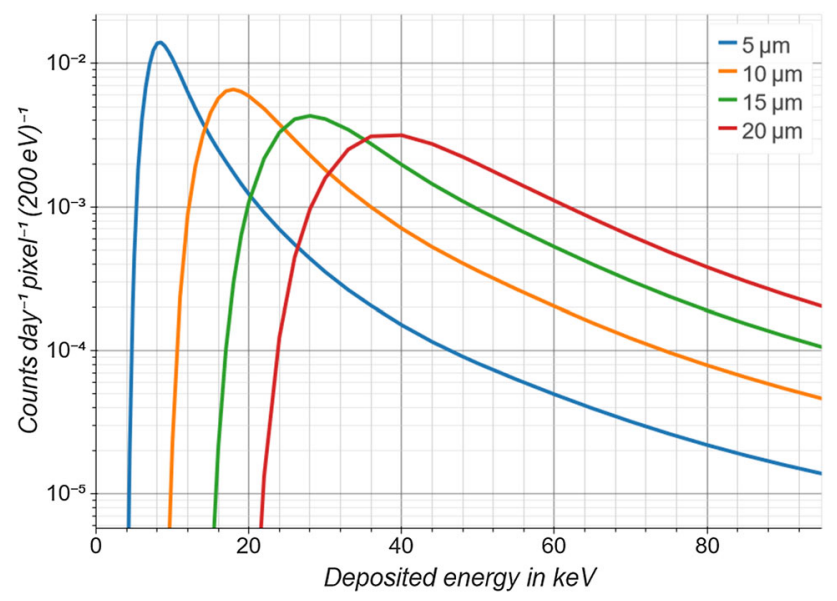

Fig. 3 Distribution of the energy deposited by muons passing gold absorbers with areas of $180 \mu \mathrm{m} \times 180 \mu \mathrm{m}$ and different thicknesses $[16-18,20]$. The fraction of muons with short path lengthes are highest for thin absorbers and therefore, the probability to deposit a small energy is largest for these thicknesses. For gold absorbers with a thickness of $10 \mu \mathrm{m}$ and lower, muons depositing less than $6 \mathrm{keV}$ could be neglected. However, by considering also resonances with the gold shells, the low energy edge could be smeared and so, more muons depositing less than $6 \mathrm{keV}$ could occur here, but should occur in the experiment. So, the probability that a muon deposits less than $4 \mathrm{keV}$ is underestimated here. Further approaches to describe the energy loss straggling in thin silicon detectors [19] show that the energy loss distribution gets smeared for lower energies by including the atomic shells in the straggling function. The same can be assumed for thin gold detectors.

\subsection{Options to recognize muon induced events}

Most likely, cosmic muons are not stopped in the absorbers, rather they also travel through the sensors of the same absorbers and through the substrate (non-active part of the chip). The pulse shape of the resulting signals will therefore be different from the signals caused by particles stopped in the absorbers. This means that these events should easily be recognized. Furthermore, a fraction of the deposited energy (heat) in the substrate could lead to a measurable increase of temperature in the neighboring pixels. This class of muon related events can be recognized via coincidence searches as well as muons only passing the substrate next to the pixels.

In addition, muons can cause secondary radiation by passing material, as for example the aluminum shield, which is directly on top of the detector array, or the substrate, which is directly below the array. The secondary particles can be created and as well deposit energy directly in the absorbers or and in the substrate. High energy secondary particles like electrons and some $\gamma$-rays ${ }^{1}$ should behave like the muons and should also easily be recognized by the resulting pulse shape of the signals and trigger time coincidences. But, low energy secondary particles like aluminum and silicon X-rays could be stopped in the absorbers. These particles could only be recognized by trigger time coincidences.

\subsection{Monte Carlo simulation}

To address these different possibilities, the effect of cosmogenic muons has been tested via Monte Carlo simulation using the version 10.06.p03 of the GEANT4 toolkit [21] considering a simplified version of the setup showed in Fig. 2 and which considers as input the energy and angular distribution of the arriving muons given in $[16,17]$. We study the effect of direct interaction of muons and of the secondary particles produced in the surrounding of the detector array.

A (primary) particle propagates a given distance (called step) in the material, which leads to total cross-sections for different types of interactions (e.g. ionization, nuclear scattering, Bremsstrahlung, etc.). A random number defines if these interactions occur in a step and if yes, the primary

\footnotetext{
$1 \gamma$-rays should mainly lose energy by Compton scattering and/or pair production and so, could deposit energy in the absorbers as well as in the substrate.
} 
Table 1 Minimum distanced produced particles have to travel in the material. If the particles have less energy, they are not produced and their energies are deposited locally. Volumes marked with (*) contain subvolumes with different production thresholds (see Fig. 4)

\begin{tabular}{ll}
\hline Volume & Min range \\
\hline MMCs & $100 \mathrm{~nm}$ \\
Thermal baths & $100 \mathrm{~nm}$ \\
$(*)$ Substrate & $1.0 \mu \mathrm{m}$ \\
SQUID chips & $1.0 \mu \mathrm{m}$ \\
Circuit board & $10 \mu \mathrm{m}$ \\
Plugs & $1.0 \mathrm{~mm}$ \\
$(*)$ Copper holder & $1.0 \mathrm{~mm}$ \\
(*)Shielding & $1.0 \mathrm{~mm}$ \\
\hline
\end{tabular}

particle loses energy and other (secondary) particles (e.g. $\delta$ rays, ${ }^{2} \mathrm{X}$-rays and Auger electrons in case of ionization) are produced if their kinetic energy are above the production energy threshold - the minimum kinetic energy, which the produced particles have to have. If the kinetic energy of the secondary particles is below the production energy cut, their kinetic energy is added to the locally deposited energy of the primary particle and these particles are not generated.

In order to study the effect of secondary particles in the detector pixels, especially low energy secondary particles, the production energy cut is set individually for each volume. This is done to save computation time and to avoid the simulation of particles which will not reach the detectors, while also considering particles with sub-keV energies. However, this production energy only affects particles generated by processes with infrared divergence, such as Bremsstrahlung and $\delta$-ray production. Particles produced by other processes, for example by radioactive decay or atomic de-excitation, are not affected by this limitation.

In the interest of clarity, not the production energy, but the equivalent, the minimum range the particles have to travel in each volume are defined (seen Table 1). In order to save computation time, the larger volumes, substrate, copper holder and shielding contain sub volumes with smaller production thresholds. These subvolumes and the production thresholds can be seen in Fig. 4, which shows the scheme of the profile of the set-up around the ECHo- $1 \mathrm{k}$ chip.

\subsection{Monte Carlo results}

$1.8 \times 10^{6}$ muons, which have to pass the ECHo-1k chip, are simulated with an angular distribution of the muon energies given by Bugaev et al. [16]. The chip is positioned such that the 72 MMCs are parallel to the ground (Fig. 2). $1.8 \times 10^{6}$

\footnotetext{
${ }^{2}$ Free electrons produced by ionization.
}

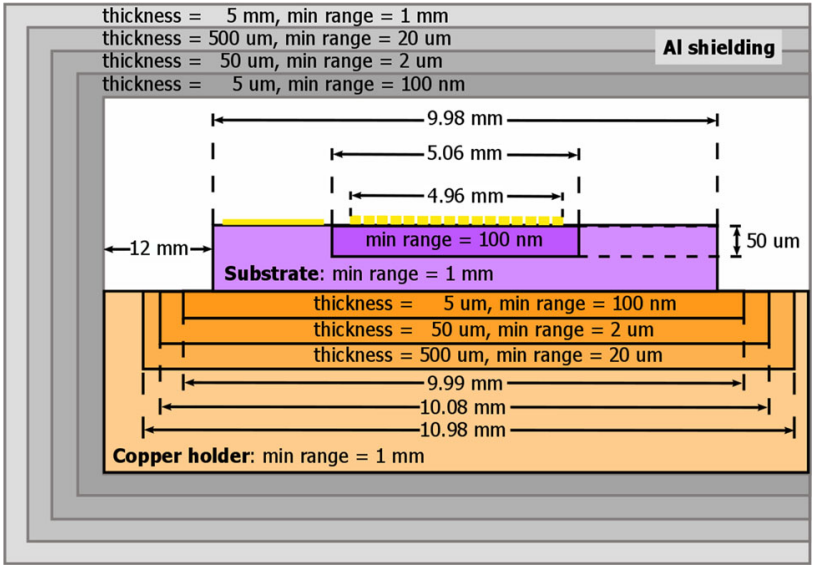

Fig. 4 Scheme of the set-up around the ECHo-1k chip (not to scale). The dimensions and production thresholds of the subvolumes of the shielding (grey), substrate (purple) and copper holder (orange) can be seen

muons is equal to about 172,521 pixel-days (2396 days, with a mean muon flux of 180 muons $\mathrm{s}^{-1} \mathrm{~m}^{-2}$ ) of measurement for the ECHo-1k array. Energies are deposited in the absorbers mostly by muons, but also secondary particles generated by muons in the surrounding materials deposit their energy in the absorbers (compare to Table 2). The major contribution is due to $\delta$-rays. Figure 5 shows the resulting summed spectrum for all pixels. Above $10 \mathrm{keV}$, the simulated spectrum is in good agreement with the Landau distribution described in [18] for an absorber thickness of $10 \mu \mathrm{m}$ (see Fig. 5a). For smaller energies, however, the spectrum differs, as expected from resonances with the atomic shell levels (Fig. 5b).

As a result of the simulation a muon induced count rate of approximately $(2.2 \pm 0.1) \times 10^{-5}$ counts day ${ }^{-1}$ pixel $^{-1}$ is expected in the region of interest defined as $10 \mathrm{eV}$ below $Q_{\mathrm{EC}}$ and less than $10^{-2}$ counts day ${ }^{-1} \mathrm{pixel}^{-1}$ in the first $4 \mathrm{keV}$, the sensitive energy range of the ECHo experiment, due to muons and their secondary particles. The muon flux can vary over time and location, thus it is also meaningful to give these numbers in relation to the muons. A muon passing the chip results in about $(2.1 \pm$ $0.1) \times 10^{-6}$ counts in the ROI or in less than $10^{-2}$ counts in the first $4 \mathrm{keV}$. Most of the particles however, are not stopped in the absorbers, which is why these events should be recognizable either by the pulse shape of the resulting signals or and by trigger time coincidences. For particles stopped in the absorbers, a signal count rate of about $(5 \pm 2) \times 10^{-7}$ counts day ${ }^{-1}$ pixel $^{-1}$ is expected in the ROI, which corresponds to $(5 \pm 2) \times 10^{-8}$ counts muon ${ }^{-1}$ (less than $10 \%$ of all muon induced events in ROI), which could only be recognized by trigger time coincidences, but in the simulation none of these events occur coincidental to other events. 
Table 2 Number of created particles in a simulation of $1.8 \times 10^{6}$ primary muons. Only particles which or whose daughter deposit energy in the absorbers are listed

\begin{tabular}{lll}
\hline$\delta$-rays produced by ionization & \\
\hline Creation volume & Mother particle & Number of $\delta$-rays \\
\hline Substrate & $\mu^{-}$ & 718 \\
Substrate & $e^{-}(\delta$-ray $)$ & 48 \\
Thermal baths & $\mu^{-}$ & 5 \\
Copper holder & $\mu^{-}$ & 351 \\
Copper holder & $e^{-}(\delta$-ray $)$ & 7 \\
Shielding & $\mu^{-}$ & 4034 \\
Shielding & $e^{-}(\delta$-ray $)$ & 153 \\
Circuit board & $\mu^{-}$ & 19 \\
Circuit board & $e^{-}(\delta$-ray $)$ & 2 \\
SQUID chip & $\mu^{-}$ & 5 \\
SQUID chip & $e^{-}(\delta$-ray $)$ & 1
\end{tabular}

Auger electrons produced by ionization

\begin{tabular}{lll}
\hline Creation volume & Mother particle & Number of augers \\
\hline Substrate & $\mu^{-}$ & 10 \\
Substrate & $e^{-}(\delta$-ray $)$ & 7 \\
Thermal baths & $\mu^{-}$ & 13 \\
Thermal baths & $e^{-}(\delta$-ray $)$ & 2 \\
Shielding & $\mu^{-}$ & 1 \\
Shielding & $e^{-}(\delta$-ray $)$ & 14
\end{tabular}

$\mathrm{X}$-rays produced by ionization

\begin{tabular}{lll}
\hline Creation volume & Mother particle & Number of X-rays \\
\hline Substrate & $e^{-(\delta \text {-ray })}$ & 2 \\
Copper holder & $e^{-(\delta \text {-ray })}$ & 1 \\
Shielding & $e^{-(\delta \text {-ray })}$ & 8 \\
\hline
\end{tabular}

Photons produced by bremsstrahlung

\begin{tabular}{lll}
\hline Creation volume & Mother particle & Number of photons \\
\hline Wafer & $e^{-(\delta \text {-ray })}$ & 28 \\
Copper holder & $e^{-(\delta \text {-ray })}$ & 81 \\
Shielding & $e^{-(\delta \text {-ray })}$ & 64 \\
\hline
\end{tabular}

Other particles

\begin{tabular}{llll}
\hline Particle & Creation volume & Process & Number \\
\hline$e^{-}$ & Copper holder & $\mu^{-} \rightarrow \mu^{-} e^{+} e^{-}$ & 1 \\
$e^{-}$ & Shielding & $\mu^{-} \rightarrow \mu^{-} e^{+} e^{-}$ & 6 \\
$e^{+}$ & Shielding & $\mu^{-} \rightarrow \mu^{-} e^{+} e^{-}$ & 5 \\
$e^{+}$ & Circuit board & $\mu^{-} \rightarrow \mu^{-} e^{+} e^{-}$ & 1 \\
$e^{+}$ & Copper holder & $\gamma \rightarrow e^{+} e^{-}$ & 1 \\
$\pi^{-}$ & Copper holder & $\mu^{-}+\mathrm{Cu}$ & 1 \\
\hline
\end{tabular}
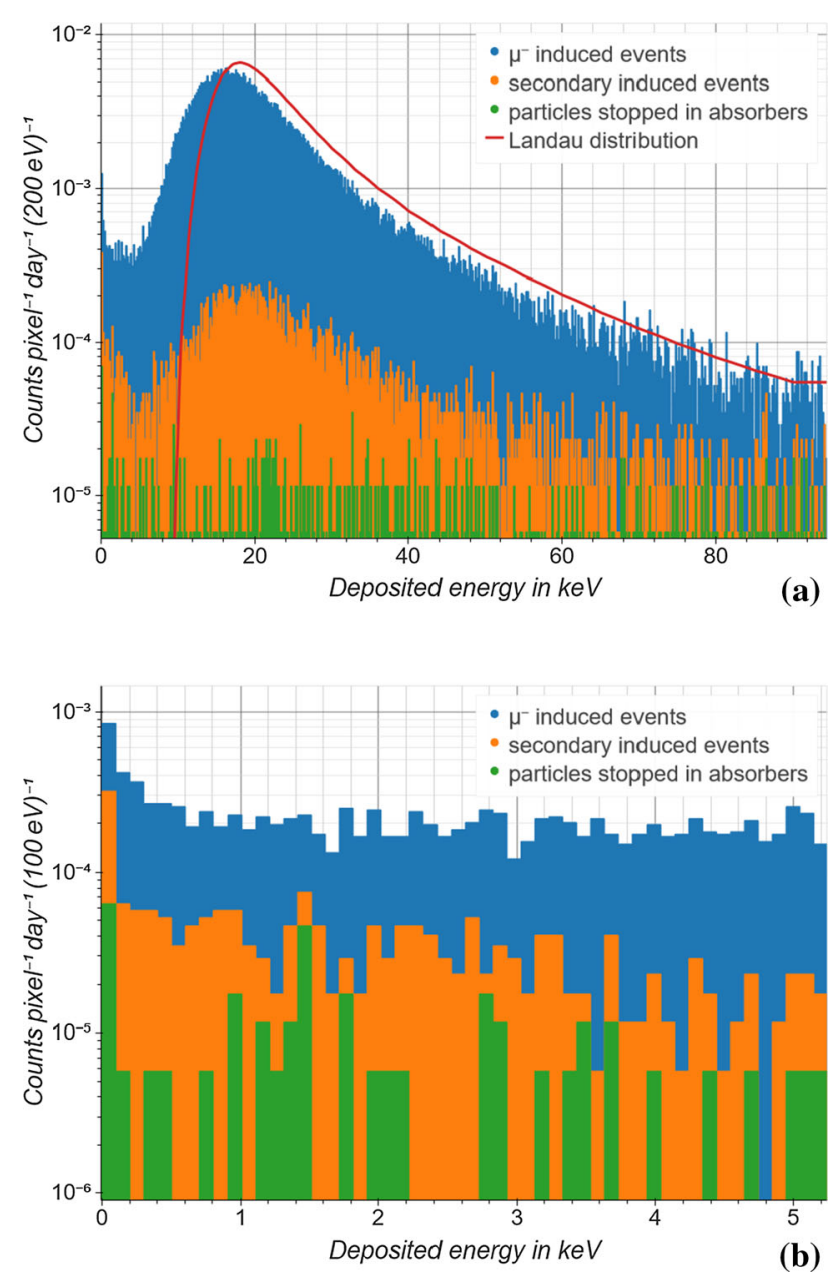

Fig. 5 a Simulated spectrum of the deposited energies in the detector pixels caused by muons (blue) and particles, mostly $\delta$-rays, generated by muons (orange). The total energy ( $\approx$ caused by muon hits) distribution follows the Landau distribution (Fig. 3) for a thickness of $10 \mu \mathrm{m}$, but only for energies larger than $10 \mathrm{keV}$. b The spectrum in the sensitive region is nearly flat

Nevertheless, the expected count rate of these events is much smaller than the expected unresolved pile-up rate, even with a muon flux as high as two times the assumed muon flux, and could be neglected. All given values only include the deposited energy by particles (muons and secondary particles) in the detector pixels directly and not events due to heat deposited into the substrate. The particles stopped in the absorbers could be coincidental with a particle hitting the substrate next to the pixels and thus, the fraction of coincidental events could be much higher than in the simulation. This class of events could not properly be studied by Monte Carlo simulations since the heat diffusion processes are not implemented in the used GEANT4 toolkit, but are discussed in Sect. 3 by interpreting the analyzed data. Direct hits in multiple pixels at the same time, caused by particles going through the absorbers, are very uncommon. In the sim- 

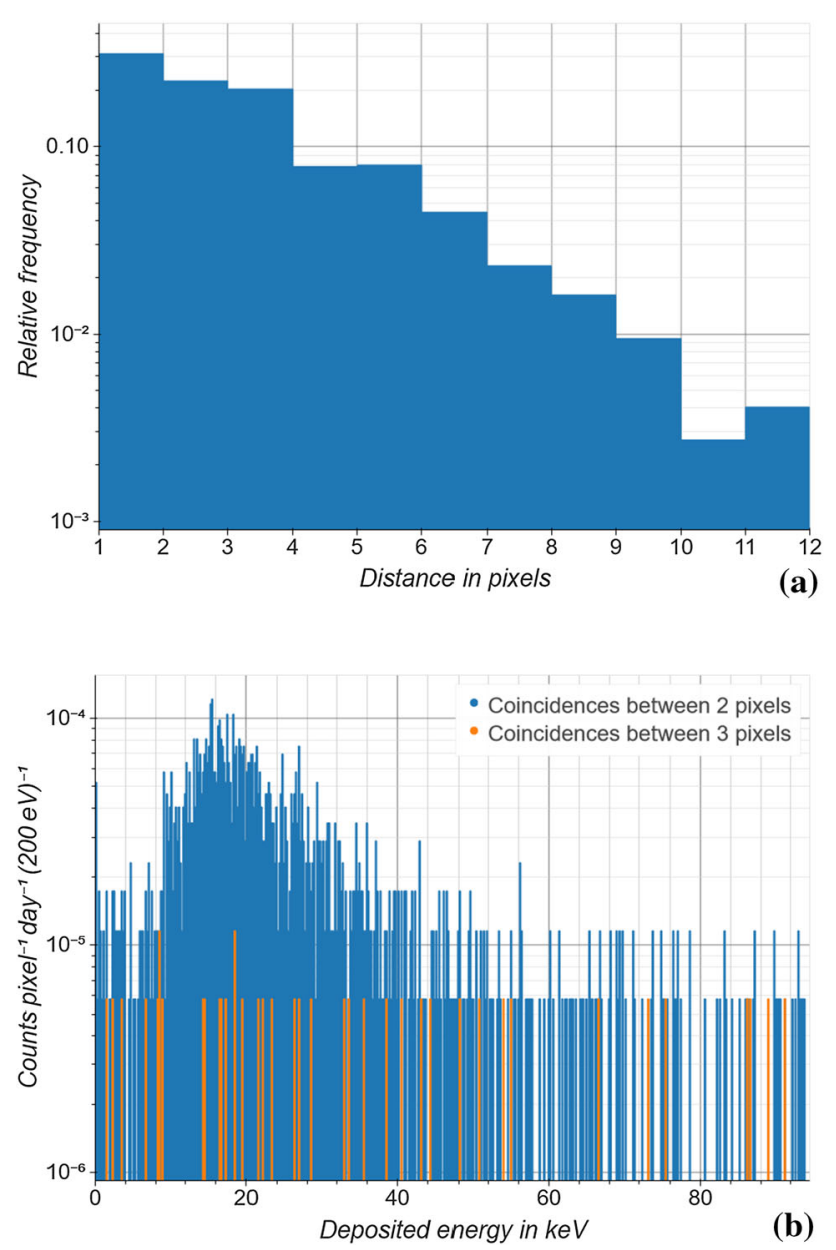

Fig. 6 a Simulated relative frequency of distances between coincidental pixels. The drop at the distance of 4 is caused due the alignment of the pixel array of $4 \times 18$ pixels. b Coincidental events of the simulated spectrum of the deposited energies in the detector pixels for a multiplicity of 2 (blue) and 3 (orange)

ulation, only about $(0.31 \pm 0.01)$ of these events (less than $1 \%$ of all occurred events in the simulation) occurred per day, from which about the half is assigned to hits of direct neighboring pixels (distance $\leq 2$ pixels, see Fig. 6 a). Direct muon hits are involved in about $81 \%$ in these events and either coincidences between two pixels $(97.7 \%)$ or between three pixels $(2.3 \%)$ are observed. As expected, the spectrum of deposited energy of coincidental events looks like the full spectrum, but with a lower count rate (compare to Fig. 6b).

\section{Measurements}

In order to investigate the influence of muons for the ECHo experiment, we report an analysis of 64 pixel-days of data, including data of four ${ }^{163} \mathrm{Ho}$ loaded pixels (= two channels) located directly next to each other. In addition, a plastic scintillator based active muon veto was operated during the 16 days of measurement. This study will also show how events caused by heat deposited in the substrate next to the pixels can be recognized by their pulse shape.

Muon veto The muon veto consists of $24130 \mathrm{~cm} \times 20 \mathrm{~cm} \times$ $2.5 \mathrm{~cm}$ sized polystyrene based scintillator panels. Each panel consists of two $125 \mathrm{~cm} \times 20 \mathrm{~cm} \times 0.8 \mathrm{~cm}$ sized plastic scintillator boards separated by a steel plate with a thickness of $0.5 \mathrm{~cm}$. Two silicon photo multipliers (SiPMs) collect the light at the ends of a u-shaped glass fiber embedded in each scintillator board. Because of the readout electronics of the SiPMs, the two scintillator boards are not completely aligned, they are misaligned by about $5 \mathrm{~cm}$. The efficiency of each panel for detecting passing muons has been tested by comparing the rates of two and three coincident muon veto panels. For this, three panels were stacked. The rate of coincidences between the top and bottom panels is then compared with the rate of coincidences between all three panels. If the detection efficiency of the panels is about $100 \%$, both rates should be the same. Otherwise, the rate of triple coincidence is a fraction of the rate of coincidences between the top and bottom panels. This measurement was performed for varying misalignment of the middle panel to determine the size of the dead zones around the corners. Resulting, the active area is about $118 \mathrm{~cm} \times 18.4 \mathrm{~cm}$ and the overall efficiency of the active area of each panel is larger than $80 \%$.

Four panels are placed at each side of the ECHo dilution refrigerator. Due to the occupied space of the refrigerator in the top region, a second layer of four panels is placed on the bottom crossed to the other four bottom panels (see Fig. 7). The muon veto raises a signal if at least two panels from two of the six veto areas, including the two bottom layers, raise a signal. A Monte Carlo simulation was performed to estimate the efficiency of the whole muon veto. A cube with an edge length of $50 \mathrm{~cm}$ is placed in the center of the veto enclosed volume, whereby the center of the cube is placed at a height of $50 \mathrm{~cm}$. The simulated muons have to pass this cube. Resulting, more than $56 \%$ of the muons passing the cube are detected by the muon veto. If only one panel has to detect a muon, the efficiency is larger than $91 \%$.

\subsection{Event classification}

As discussed in Sect. 2.2, we expect different classes of events in MMCs arrays related to cosmic muons. In the following, we present a pulse shape analysis method, developed to study these. The aim of this analysis is to identify possible deviations on the signal shape with respect to the signal shape due to the decay of ${ }^{163} \mathrm{Ho}$ in MMC absorbers. This will 


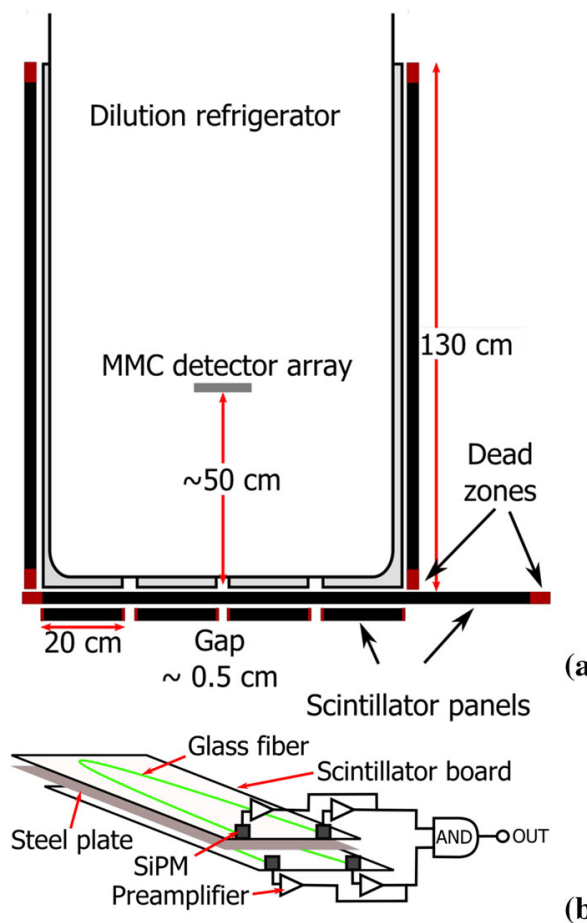

(b)

Fig. 7 a Section of the muon veto surrounding the ECHo dilution refrigerator. Four of 24 polystyrene based scintillator panels (shown in black) are placed at each side of the refrigerator (shown in white). A second layer of 4 panels are placed on the bottom crossed to the other bottom panels to compensate the missing layer on top of the cryostat. b A panel consists of two scintillator boards separated by a steel plate. The produced light is collected by an embedded u-shaped glass fiber and read out by two SiPMs at each end of the glass fiber

be helpful to determine strategies to identify muon related events, which do not cause any coincidence among the pixels. This pulse shape analysis method is based on the determination of the energy relation to a signal using different algorithms and combine the different information. The analyzed signals correspond to time traces of about $2 \mathrm{~ms}$ length (= $2^{14}$ samples) of which the first third corresponds to the pre-trigger and the last two thirds to the actual triggered signal. When talking about pulses in the following, the last two third of the time traces (without the pre-trigger) are considered.

Template fit The average of one to five thousand pulses with amplitudes corresponding to energies of the Ho-MI line is calculated to obtain a template pulse for each pixel and measurement day (see the blue curve in Fig. 8). In order to shorten the pile-up window, only the first third of the template (the orange part of the template) is fitted to each pulse using the $\chi^{2}$ method of varying the amplitude of the template. To obtain higher sensitivity for pileup in the first third of the pulses, in addition the template's tail, the last quarter (of the first third, the green dotted part), is fitted to each signal. The goodness of the fit is

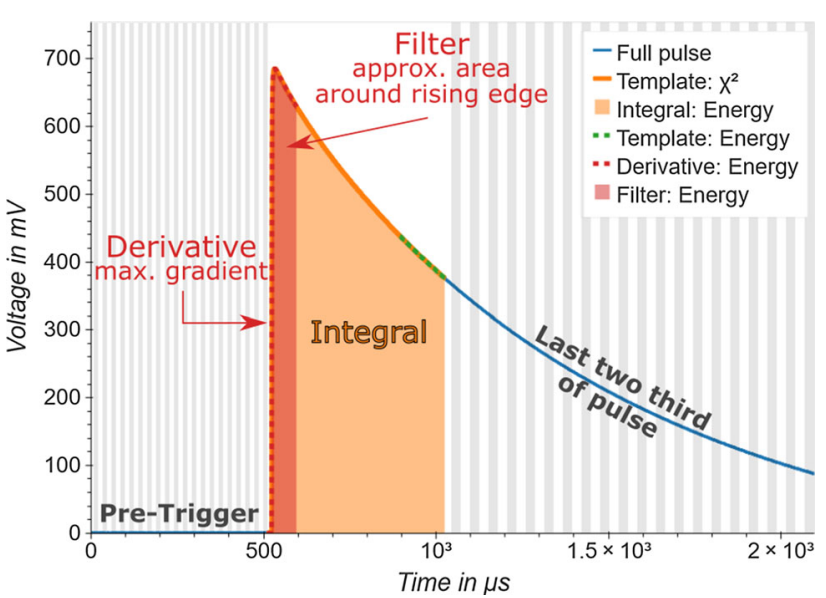

Fig. 8 The parts of the time signal used in the different analysis methods are indicated in different colors. A template pulse is shown in blue. The first third after the pre-trigger (orange line) is fitted to all pulses in order to obtain the reduced $\chi^{2}$. In addition the tail of this part of the template (the last quarter, green dotted) is fitted to all pulses to obtain the amplitude of the fit to increase the sensitivity to pile-up occurring in the first third of the pulses. The first part of template around the rising edge (red dotted line) is convoluted with each pulse, which mainly results in the area under the pulse around the rising edge (red area). The derivative of the smoothed pulses are calculated to obtain the maximum gradient. The area under the pulses (orange area) are calculated to identify pile-up pulses

given by the reduced $\chi^{2}$ (i.e. $\chi^{2} \mathrm{dof}^{-1}$ ) and is determined by fitting the first third part to the pulses. The amplitude $A_{\text {Template }}$ of the fit obtained from fitting the tail is proportional to the deposited energy $E$, which results in the reconstructed energy $E_{\text {Template }}$. This method is sensitive to the overall pulse shape, but mainly to falling edge of the pulses.

Matched filter A time trace with the length of four times the distance between the maximum of the template pulse and the beginning of the template pulse is built. For this a short length is chosen, so that this method is insensitive to pile-up occurring in the first third of the pulse. Each pulse is convoluted with this time trace (so called matched filtering). The amplitude of the response function $A_{\text {Filter }}$ describes mainly the area below the pulse around the rising edge and results in the reconstructed

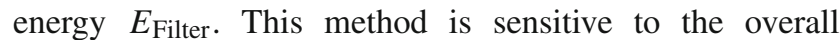
pulse shape around the rising edge (see the red area in Fig. 8).

Derivative The pulses are smoothed by using a moving average filter. The maximum of the derivative of the smoothed pulses $A_{\text {Derivative }}$ results in the reconstructed energy $E_{\text {Derivative }}$ (see the red curve in Fig. 8). This method is sensitive to pile-up with short time differences and to other effects, which could change the rise time. 


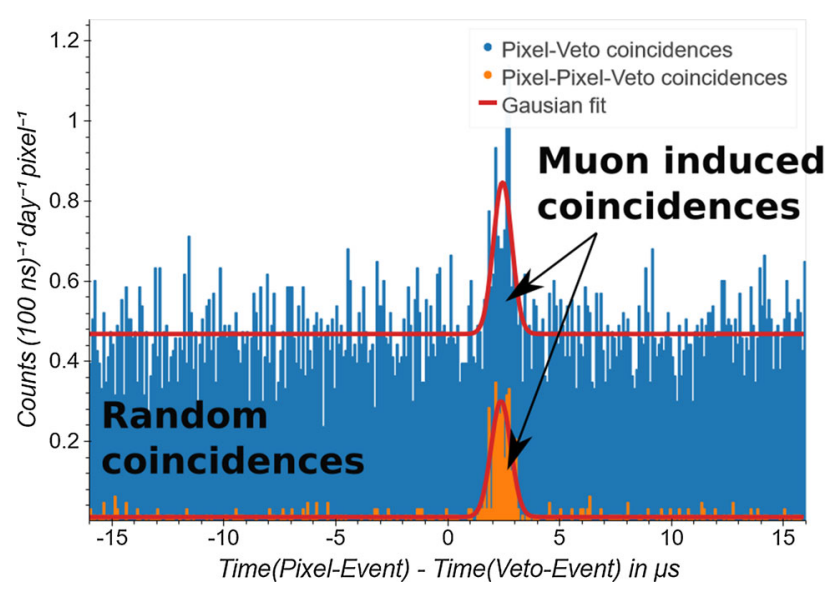

Fig. 9 The time difference $\delta t$ between the pixel events and muon veto events $\delta t=t$ (pixel event) $-t$ (veto event). The events shown in blue are all pixel events coincidental to the muon veto and the events, which are in addition coincidental to other pixel events are showed in orange. A peak at $\delta t \approx 3 \mu \mathrm{s}$ is visible in both. The flat spectrum corresponds to random coincidences between pixel events and muon veto, the events in the peak are induced by muons, which cross the muon veto and interact with the MMCs

Integral The area $A_{\text {Integral }}$ under the first third part of the pulses is calculated and results in the reconstructed energy $E_{\text {Integral }}$. This method is sensitive to pile-up with large time differences and to other effects which could change the mean decay time (see the orange area in Fig. 8 .

All four methods are sensitive to the pulse shape but in different ways. The ratio of the reconstructed energies is the same for all energies, but is different for different kinds of signals. The following analysis of muon related events is performed by identifying signals caused by particles, for which the energy of a particle is fully thermalized in the MMC absorbers, meaning the particle does not propagate through the MMC sensor and or the substrate, such as the products of the ${ }^{163} \mathrm{Ho}$ EC decay. These type of signals will be called full-thermalization signals in the following. Muon related signals should mostly be caused by particles which are not fully stopped in the absorbers or signals due to heat propagating from the substrate to the MMCs. Thus, pulses caused by muons should differ from the full-thermalization pulses.

For all full-thermalization pulses the ratio of the reconstructed energies is set to 1 . The same is done for the reduced $\chi^{2}$, where the full-thermalization pulses are located around $\log \left(\chi^{2} \operatorname{dof}^{-1}\right) \simeq 0$.

\subsection{Muon induced events}

The muon coincidences, also called Pixel-Veto coincidences in the following, can be identified by histogramming the time difference of the detector pixel events to all events of the muon veto: $\delta t=t$ (pixel event) $-t$ (veto event). Figure 9 shows the histogram of the time delays for all measured pixel events with $|\delta t| \leq 16 \mu \mathrm{s}$. The blue events corresponds to muon veto coincidences with at least one pixel event and the orange events correspond to muon veto coincidences with more than one pixel events. A peak at $\delta t \approx 2.5 \mu \mathrm{s}$ is visible on both. ${ }^{3}$ These are events corresponding to muons crossing the muon veto and interacting with the detector pixels. The time shift of $2.5 \mu \mathrm{s}$ is caused by the different delays in the readout chains of the ECHo detector and the veto. The random coincidences can be seen as a flat background.

The number of muon induced Pixel-Veto coincidence events (the blue colored data in Fig. 9) can be determined by fitting a Gaussian,

$n(t, \delta t, a, \sigma, c)=a \cdot \exp \left(-\frac{(t-\delta t)^{2}}{2 \sigma^{2}}\right)+c$,

to the time difference histogram. After subtracting a flat background rate of $(0.468 \pm 0.005)$ counts $(100 \mathrm{~ns})^{-1}$ day $^{-1}$ pixel $^{-1}$, the sum of the binned raw number of counts $n_{i}$ in the $3 \sigma$ range around $\delta t=(2.45 \pm 0.05) \mu \mathrm{s}$

$N=\sum_{i=1.3 \mu \mathrm{s}}^{3.6 \mu \mathrm{s}} n_{i}-c$

is $N=242 \pm 20$ counts, with $\sigma=(410 \pm 50)$ ns. $N$ is calculated with varying parameters to estimate the errors. The peak position is shifted by $\delta_{\delta t}=50 \mathrm{~ns}$, the variance is reduced/increased by $3 \cdot \delta_{\delta \sigma}=150 \mathrm{~ns}$ and the background is shifted by $\delta_{c}=0.005$ counts $(100 \mathrm{~ns})^{-1}$ day $^{-1}$ pixel $^{-1}$ :

$N_{\delta t}^{+}=\sum_{i=1.3 \mu \mathrm{s}}^{3.7 \mu \mathrm{s}} n_{i}-c, \quad N_{\delta t}^{-}=\sum_{i=1.2 \mu \mathrm{s}}^{3.6 \mu \mathrm{s}} n_{i}-c$,

$N_{\sigma}^{+}=\sum_{i=0.8 \mu \mathrm{s}}^{4.1 \mu \mathrm{s}} n_{i}-c, \quad N_{\sigma}^{-}=\sum_{i=1.7 \mu \mathrm{s}}^{3.2 \mu \mathrm{s}} n_{i}-c$,

$N_{c}^{ \pm}=\sum_{i=1.3 \mu \mathrm{s}}^{3.6 \mu \mathrm{s}} n_{i}-c \pm \delta_{c}$,

where $\delta_{i}$ are the variances of the best fit parameters. Further, the error $\delta_{N}$ for $N$ is calculated with

$\delta_{N}=\operatorname{sqrt}\left(\sqrt{N}^{2}+\sum_{i, \pm}\left(N_{i}^{ \pm}-N\right)^{2}\right)$.

\footnotetext{
3 The time shift appears because of the two different readout chains of the muon veto and of the MMC array.
} 
This is in very good agreement with the integral of the fit in the $3 \sigma$ range,

$$
\begin{aligned}
R & =\int_{\delta t-3 \sigma}^{\delta t+3 \sigma} a \cdot \exp \left(-\frac{(t-\delta t)^{2}}{2 \sigma^{2}}\right) \mathrm{d} t \\
& =a \cdot \sqrt{2 \pi} \cdot \operatorname{erf}\left(\frac{3}{\sqrt{2}}\right) \cdot \sigma,
\end{aligned}
$$

with the error function

$$
\operatorname{erf}(x)=\frac{2}{\sqrt{\pi}} \int_{0}^{x} \exp \left(-t^{2}\right) \mathrm{d} t .
$$

This integral results in $247 \pm 28$ counts and the error is calculated using the Gaussian error propagation and the variances of the best fit parameters of $\sigma$ and $a$,

$\delta_{R}=\sqrt{2 \pi} \cdot \operatorname{erf}\left(\frac{3}{\sqrt{2}}\right) \cdot \sqrt{\left(\sigma \delta_{a}\right)^{2}+\left(a \delta_{\sigma}\right)^{2}}$.

In prior sections we estimated a total veto muon detection efficiency of about $56 \%$. While this efficiency needs independent verification, it presents a consistent picture of our experimental set-up. In that case, we infer an estimated rate of approximately $(6.9 \pm 0.5)$ counts day ${ }^{-1}$ pixel $^{-1}$. We note the muon rate may vary over time and location, thus the exact absolute rate should be determined for the specific experimental conditions. However, we would like to note that under the consistency of these assumptions, the current set-up's measurements suggest an effective area of about 14 times the pixel area, if we also assume an approximate average muon flux of 180 muons s $\mathrm{s}^{-1} \mathrm{~m}^{-2}$ [1] $\left(=0.5\right.$ muons day ${ }^{-1}$ pixel $\left.^{-1}\right)$. These signals could either be caused by muons or secondary particles passing through an absorber, the corresponding sensor and the substrate next to the pixels, or muons and secondaries passing only the substrate next to the pixels. Both classes are defined as substrate events in the following, where heat could diffuse through the substrate to the pixels. For these events, a single muon passing through the chip should be detected by more than one pixel.

For these reasons we study events that are coincident with the muon veto and in addition to other pixels $(\mid t$ (pixel event $\mathrm{A})-t$ (veto event $\mathrm{B}) \mid \leq 4 \mu \mathrm{s})$, called PixelPixel-Veto coincidences in the following. Those events are indicated by the orange histogram in Fig. 9 and result in $194 \pm 12$ counts in the $3 \sigma$ range leading to a count rate of $(5.5 \pm 0.3)$ counts day ${ }^{-1}$ pixel $^{-1}$, which is also in agreement with the integral of the fit in the same region resulting in a rate of $(5.7 \pm 0.2)$ counts day ${ }^{-1}$ pixel $^{-1}$ or $202 \pm 8$ counts. By considering the count rate with the Pixel-Pixel-Veto multiplicity, about 10 muons day ${ }^{-1}$ per active area were detected, which corresponds to a muon flux for an area equal to 5 times the active area.

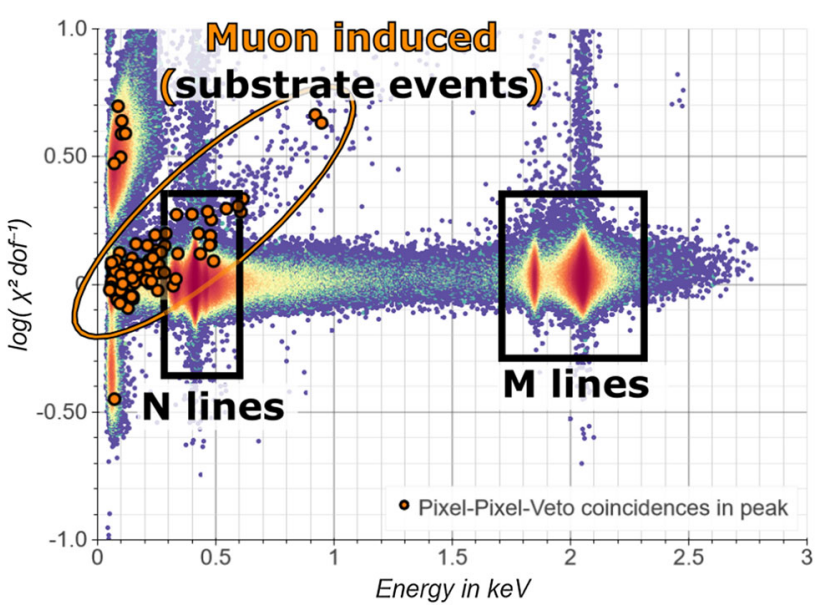

Fig. 10 The reduced $\chi^{2}$ plotted against $E_{\text {Filter }}$. The orange dots are the muon veto coincidences, which are in addition coincident to other pixel events. The ${ }^{163} \mathrm{Ho}$ EC lines are visible. The muon induced substrate events (true coincidences) have mostly low energies $(E<500 \mathrm{eV})$ and a low reduced $\chi^{2}<10$

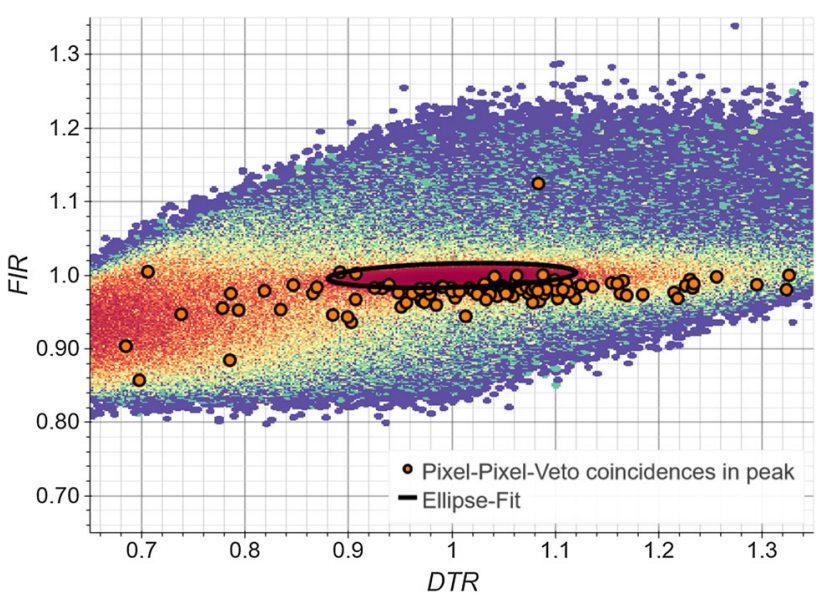

Fig. 11 The TFR parameter against the DTR parameter. The orange dots are the muon induced signals, mostly located in the surrounding of the ellipse. The pulses caused by the decay of ${ }^{163} \mathrm{Ho}$ are located inside the ellipse

Substrate events can be discriminated from the other events by their pulse shape, so that substrate events not recognized by the coincidence method can be identified. Muon induced events, extracted using the information of the time delay with the muon veto signal and the coincidences between pixel events - the events of the orange histogram of Fig. 9 in the $3 \sigma$ range - are highlighted (orange dots) in the scatter plot of the reduced $\chi^{2}$ and the energy $E_{\text {Filter }}$ (Fig. 10). This sample of events coincidental to the muon veto is used because of the low fraction of random coincidences, which implicates a high fraction of muon induced events. However, not all muon induced events have to generate coincidences among pixels. In the scatter plot in Fig. 10 also the populations of events due to the EC in 


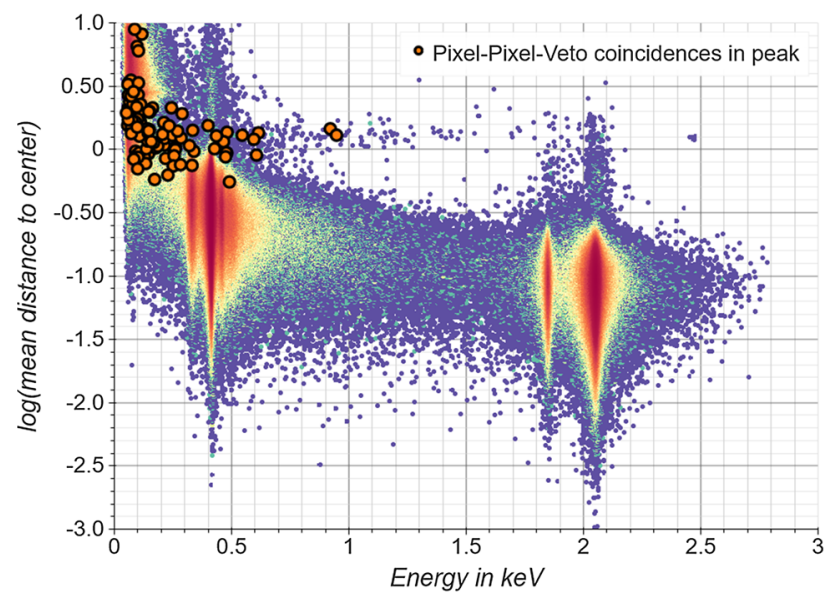

Fig. 12 The logarithm of the mean distance to the ellipses' centers against $E_{\text {Filter }}$. The orange dots are the muon veto coincidences, which are in addition coincident to other pixel events, located in a band around mean distance $=1$. Pulses due to the EC in ${ }^{163}$ Ho show smaller distances

${ }^{163} \mathrm{Ho}$ are well visible, the M-lines around $2 \mathrm{keV}$ and the $\mathrm{N}$-lines around $400 \mathrm{eV}$. The muon induced events overlap strongly with the full-thermalization pulses $\left(\chi^{2} \mathrm{dof}^{-1}<10\right)$ in the scatter plot for energies less than $1 \mathrm{keV}$. By considering the pulse shape parameters (PSPs) FIR $=\frac{E_{\text {Filter }}}{E_{\text {Integral }}}$ versus DTR $=\frac{E_{\text {Derivative }}}{E_{\text {Template }}}$, the pulse shape discrimination can be improved (see Fig. 11). As expected, in this parameter space a high density can be seen in a region with an ellipse shape, whose size will discussed later, with an center at around FIR $=$ DTR $=1$. Pulses located in this region can be assigned to the class of full-thermalization pulses and muon induced substrate events are mostly located outside the ellipse.

With the parameters defined in Sect. 3.1 six ratios (= pulse shape parameters) can be defined: FIR, DTR, TIR $=\frac{E_{\text {Template }}}{E_{\text {Integral }}}$, $\mathrm{DFR}=\frac{E_{\text {Derivative }}}{E_{\text {Filter }}}, \mathrm{DIR}=\frac{E_{\text {Derivative }}}{E_{\text {Integral }}}$ and TFR $=\frac{E_{\text {Template }}}{E_{\text {Filter }}}$. By plotting these PSPs against each other, the region of fullthermalization pulses can be investigated in different parameter spaces. Useful parameter spaces are FIR against DTR, TIR against DFR and DIR against TFR. ${ }^{4}$ An ellipse can be fitted to each of these three scatter plots and stretched, such that the ratio of the amplitudes of the ${ }^{163} \mathrm{Ho} \mathrm{MI}$ and NI lines is equal to the theoretical ratio. It has to be noted that for this analysis a perfect knowledge of the ${ }^{163} \mathrm{Ho} \mathrm{EC}$ spectrum is assumed, because the ratio of the amplitudes depends on $Q_{\mathrm{EC}}$ and the neutrino mass, but a non-zero neutrino mass allowable by current experimental evidence has only a marginal effect on this analysis. From these three ellipses, a mean distance to the ellipses' centers, while weighting the PSPs with the half axes of the ellipses, can be calculated. The corre-

\footnotetext{
$\overline{4}$ These scatter plots are shown in the appendix, as well as scatter plots of parameter spaces which are not useful for this analysis.
}
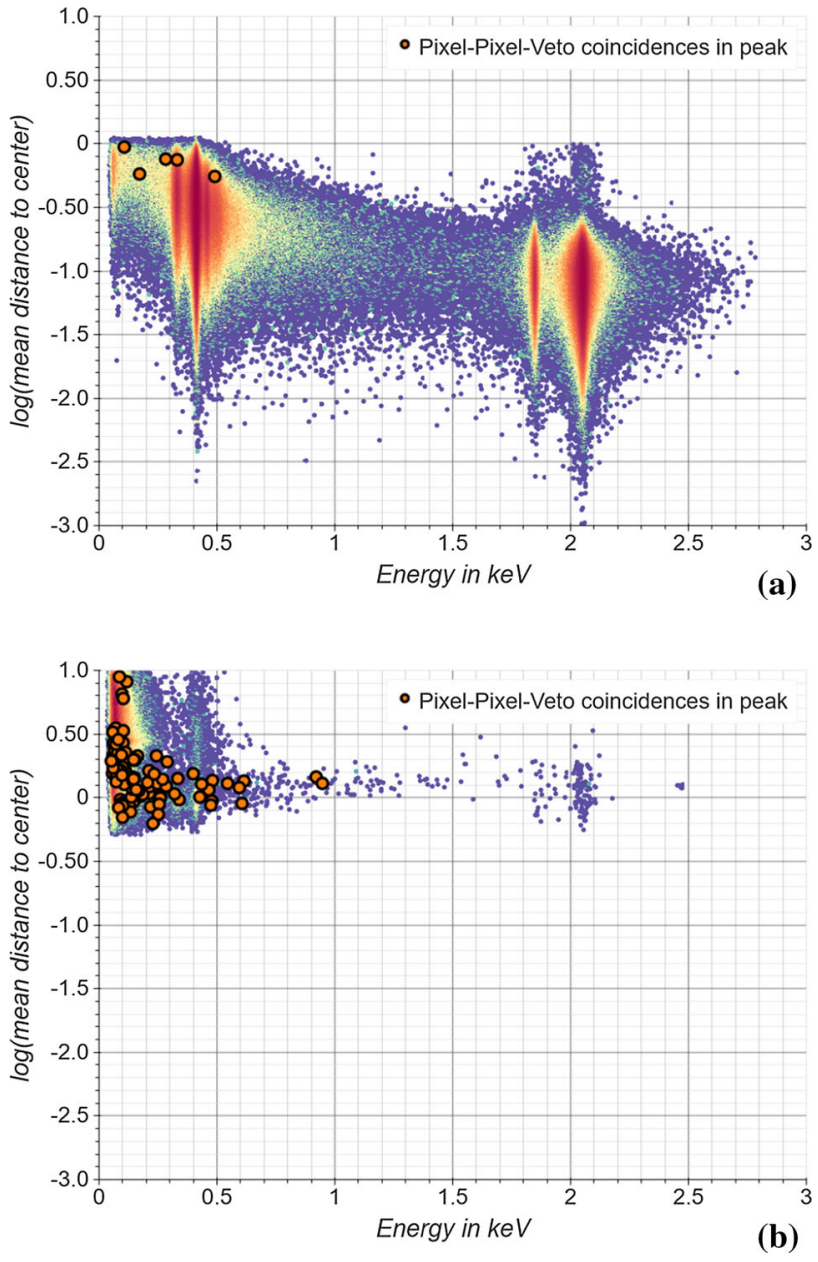

Fig. 13 The logarithm of the mean distance to the ellipses' centers against $E_{\text {Filter }}$ a Only pulses, which are located in all three ellipses are shown. The band around distance $\approx 1$ with the substrate events inside disappeared. Mainly the pulses due to the EC in ${ }^{163} \mathrm{Ho}$ remain. b Events, which are at least not located in one of the three ellipses are shown. Mainly the band around distance $\approx 1$ remains, which contains among other things of (muon induced) substrate events

sponding scatter plot of the mean distance to the center and the deposited energy can be seen in Fig. 12. Two bands are visible. One band containing ${ }^{163} \mathrm{Ho}$ EC events shows decreasing distances (mean distance $<1$ ) for increasing energies, meaning that these events are located inside the three ellipses. The second band at a mean distance $\approx 1$ contains triggered noise, GSM induced signals and is populated with (muon induced) substrate events. Due to the energy dependence of this parameter, also a fraction of the ${ }^{163} \mathrm{Ho}$ EC O-lines is cut. In order to minimize or remove the energy dependency, different approaches are under development. If a pulse shows a mean distance of 1 , this pulse is on average located at the edge of the ellipses. By defining a cut, such that pulses have to be located in all three ellipses, called ellipse cut in the following, most of the ${ }^{163} \mathrm{Ho}$ EC events can be separated from the substrate events (compare to Fig. 13). 

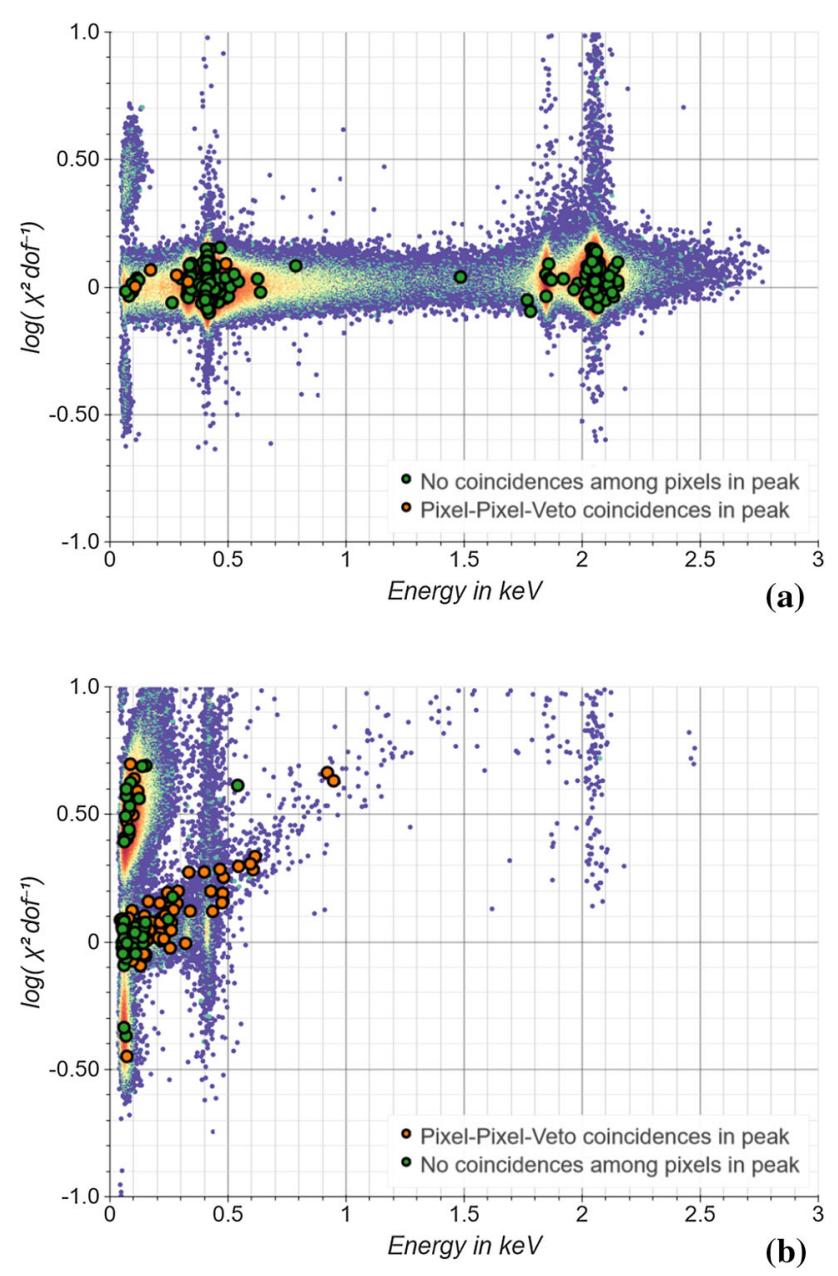

Fig. 14 The reduced $\chi^{2}-E_{\text {Filter }}$ scatter plot. The green dots are events coincidental to the muon veto but not coincidental to other pixel events and the orange dots are Pixel-Pixel-Veto coincidences. Random coincidences between the muon veto and pixel events can mostly be found in data only coincidental to the muon veto and (muon induced) substrate events generate mostly coincidences among pixels. a Events located inside of all three ellipses are shown. The ${ }^{163} \mathrm{Ho}$ EC events with the M and $\mathrm{N}$ lines can be identified. $\mathbf{b}$ Events with a value located outside at least of one ellipse

Figure 14a shows all events located inside each of the three ellipses plotted in the parameter space $\chi^{2}$ dof $^{-1}$ versus $E_{\text {Filter }}$. Likewise, Fig. 14b shows all events, which have at least one value outside of an ellipse. The muon related events present in Fig. 14a correspond to random coincidences between the muon veto and one or more of the ECHo- $1 \mathrm{k}$ pixels. The total number of these events is 757 , which is a bit lower than the prediction of the flat distribution in Fig. 9 of $781 \pm 8$ counts. The events, which are selected using the ellipse cut and shown in Fig. 14b, can be related to triggered noise, pile-up events and clearly events related to the passage of muons through the chip. The number of these events is 269 and agrees well with the number obtained by the Gaussian fit in Fig. 9 of $242 \pm 20$. Events belonging to the last category are located mostly at reconstructed ener-

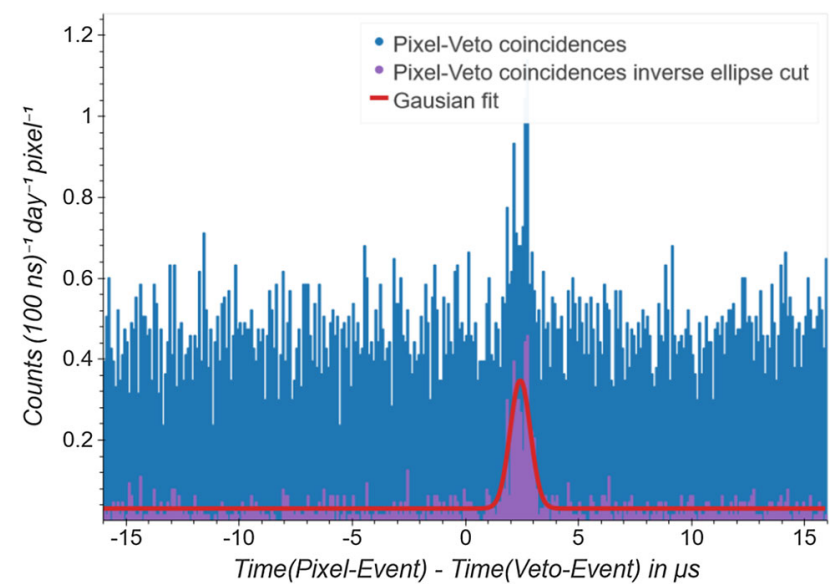

Fig. 15 The time difference $\delta t$ between the pixel events and muon veto events $\delta t=t$ (pixel event) $-t$ (veto event). The events showed in blue are all pixel events. The fraction, which are located outside of at least one of the three ellipses is shown in purple. The amount of random coincidences is clearly reduced. And (muon induced) substrate events are located outside of the ellipses

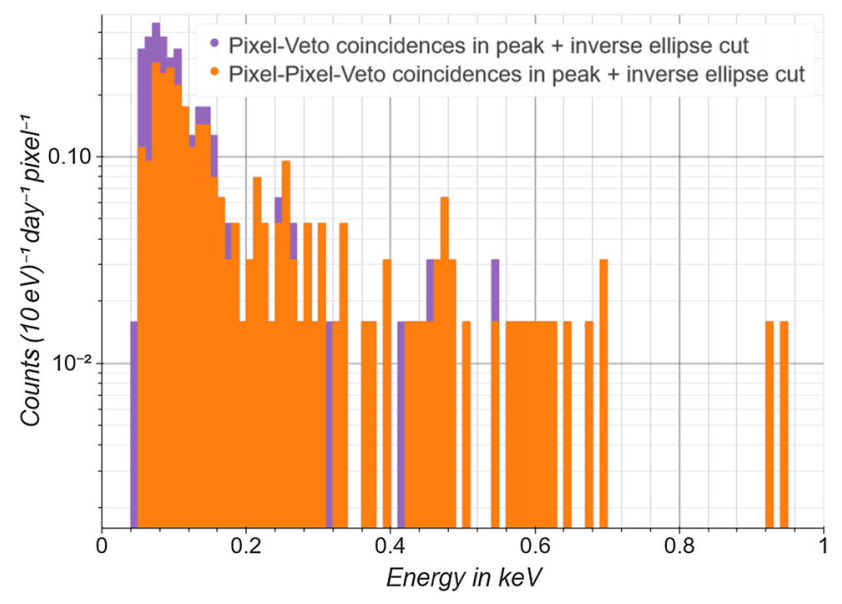

Fig. 16 The energy spectrum of the muon induced events. No features can be recognized. The highest measured energy is about $950 \mathrm{eV}$ up to now, what is far below the Q-value of the ${ }^{163} \mathrm{Ho} \mathrm{EC}$

gies below $1 \mathrm{keV}$ and $\chi^{2} \operatorname{dof}^{-1}<10$. For (muon induced) substrate events with energies less than $1 \mathrm{keV}$ a pure $\chi^{2}$ cut would not have been enough to identify them, while the use of the ellipse cut can identify and remove them from the spectrum.

By fitting a Gaussian to the time difference distribution of coincidences between pixel events and the muon veto - the blue events of Fig. 9, which are located outside of the ellipses, about $215 \pm 15$ events are selected (see Fig. 15), which results in a count rate of $(6.1 \pm$ 0.5 ) counts day ${ }^{-1}$ pixel $^{-1}$ and the integral of the fit in the $3 \sigma$ range results in $(6.3 \pm 0.2)$ counts day ${ }^{-1}$ pixel $^{-1}$ or $222 \pm 8$ events. These rates are similar to the ones taken from the fits of full $\delta t$-spectra and the spectra corresponding 
to coincidences, which are in addition coincidental to other pixel events.

Looking at the deposited energy spectrum of these muon induced substrate events (see Fig. 16), one finds the highest measured muon induced event at an energy of about $950 \mathrm{eV}$, which is far below the ${ }^{163} \mathrm{Ho}$ EC spectrum endpoint of $Q_{\mathrm{EC}} \simeq 2800 \mathrm{eV}$. From the simulation of muons passing the detector (Fig. 5b), where substrate hits are not included, only less than 0.6 direct hits of muons and secondary particles are expected in 64 pixel-days within 0$4 \mathrm{keV}$. Compared with about 215 measured muon induced substrate events in 64 pixel-days, which can be caused by muons depositing energies in the substrate, it can be concluded that this kind of events occurs with a much higher frequency than direct hits. To determine the rate of muon induced events in the region of the Ho EC endpoint, we extrapolate the observed muon induced spectrum conservatively by fitting a flat distribution to the last $400 \mathrm{eV}$ (from $600 \mathrm{eV}$ to $1 \mathrm{keV}$ ), which result in a ratio reduction of about $1.2 \times 10^{-3}$, or $(7 \pm 2) \times 10^{-3}$ counts day ${ }^{-1}$ pixel $^{-1}$ in the ROI under our assumed veto efficiency. The error is given by the variance of the single fit parameter. In this conservative scenario, these events are recognized not only by the coincidence with the muon veto, but also by pulse shape analysis and or coincidence among MMC channels. Due to the multiple ways of recognizing muon related events and to the fact that these events are mainly located at energies less than $1 \mathrm{keV}$, we can conclude that this background will not be dominant at the end point region of the ${ }^{163}$ Ho EC spectrum.

\section{Conclusion}

The goal of the ECHo collaboration is to reduce all background contributions in the experiment, so that the unresolved pile-up, with an estimated count rate of $2 \times$ $10^{-4}$ counts day ${ }^{-1}$ pixel $^{-1}$ in the last $10 \mathrm{eV}$ below $Q_{\mathrm{EC}}$, remains the dominant background contribution.

The simulation of muons and secondary particles produced by muons passing the detectors (called direct hits) predict a rate of $2 \times 10^{-5}$ counts day ${ }^{-1}$ pixel $^{-1}$ in the ROI, which is less than the unresolved pile-up rate for an activity of $10 \mathrm{~Bq}$ of ${ }^{163} \mathrm{Ho}$ per pixel. More than $90 \%$ of these events should be recognizable by time trigger coincidences and by the pulse shape analysis. Particles hitting the substrate next to the detector-pixels can also cause a signal in the detector. These events can not be simulated within the GEANT4 toolkit, but can be studied with measured muon induced substrate events. We found that (muon induced) substrate events can easily be recognized by pulse shape analysis.
From our analysis, we were able to infer a muon induced substrate event rejection rate of about $1.2 \times 10^{-3}$. Under the assumption of our muon veto detection efficiency, this equates to about 6 muon induced substrate events day ${ }^{-1}$ pixel $^{-1}$ being observed from which $(7 \pm 2) \times$ $10^{-3}$ counts day $^{-1}$ pixel $^{-1}$ are located in the ROI. We are able to recognize these events by searches for coincidence among pixels, by the use of an active muon veto and by a pulse shape analysis. For this, three methods, the analysis of the muon veto coincidences with one channel, the analysis of the veto coincidences with more than one channel and the analysis of pulse shape parameters are discussed and result in similar count rates of muon induced events. Most muon events are caused by energy deposition in the substrate around the MMC array and can very well be recognized by the induced pulse shape and typically also by coincidence. This shows that substrate events, independent from their origin, can be discriminated from ${ }^{163}$ Ho EC-like events by the use of both methods. In the future the veto efficiency can be increased, if the veto condition of at least two panels have to detect a muon is switched to at least one panel detects a muon.

Acknowledgements The experiment described in this paper was possible thanks to the DFG Research Unit FOR 2202 Neutrino Mass Determination by Electron Capture in $163 \mathrm{Ho}$, ECHo (funding under JO 451/12, GA 2219/2-2). We acknowledge the support of the clean room team of the Kirchhoff-Institute for Physics, Heidelberg University. F. Mantegazzini acknowledge support by the Research Training GroupHighRR (GRK 2058) funded through the Deutsche Forschungsgemeinschaft, DFG.

Data Availability Statement This manuscript has no associated data or the data will not be deposited. [Authors' comment: The data sets used and generated by the analysis are available from the corresponding author on reasonable request.]

Open Access This article is licensed under a Creative Commons Attribution 4.0 International License, which permits use, sharing, adaptation, distribution and reproduction in any medium or format, as long as you give appropriate credit to the original author(s) and the source, provide a link to the Creative Commons licence, and indicate if changes were made. The images or other third party material in this article are included in the article's Creative Commons licence, unless indicated otherwise in a credit line to the material. If material is not included in the article's Creative Commons licence and your intended use is not permitted by statutory regulation or exceeds the permitted use, you will need to obtain permission directly from the copyright holder. To view a copy of this licence, visit http://creativecomm ons.org/licenses/by/4.0/.

Funded by $\mathrm{SCOAP}^{3}$. 


\section{Appendix}

The parameter spaces are chosen so that no obvious correlations between the parameters are visible like in example of the parameter spaces shown in Fig. 17 and Fig. 18.
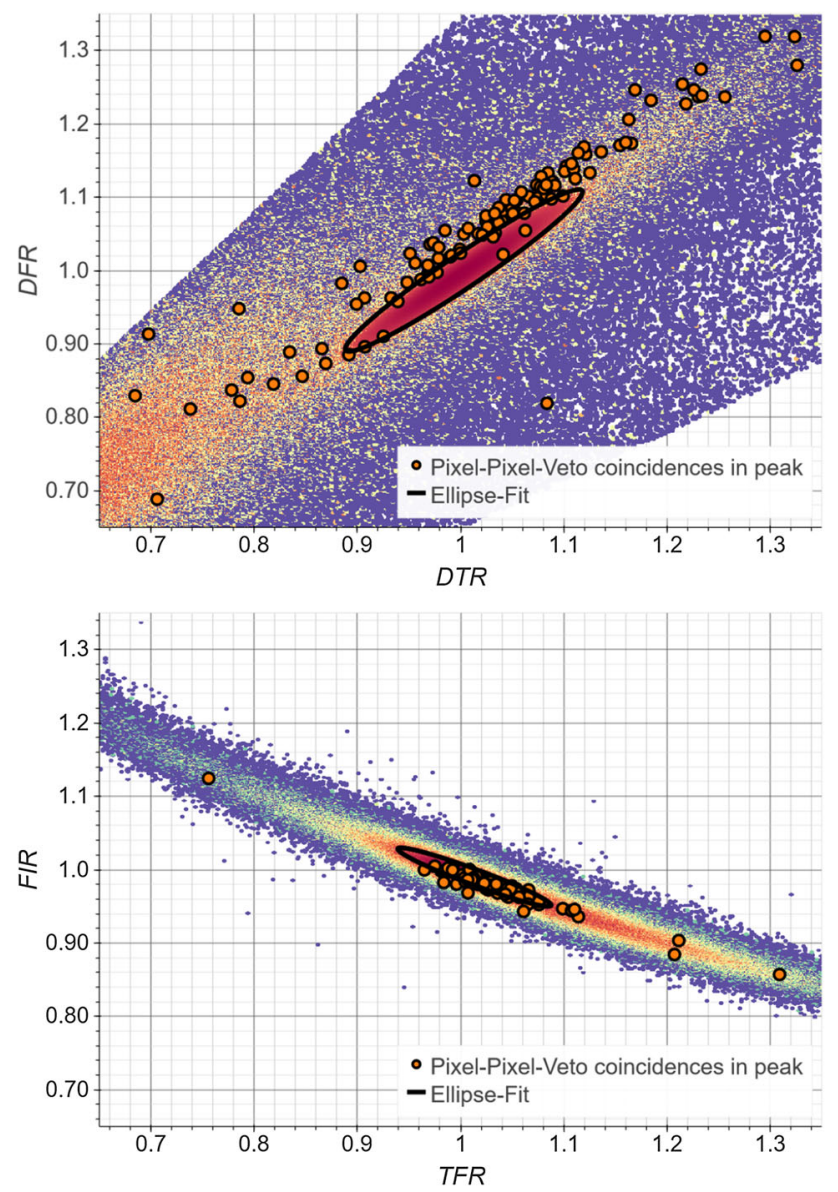

Fig. 17 Sample of non useful parameter spaces. It is clearly visible that these parameters are correlated
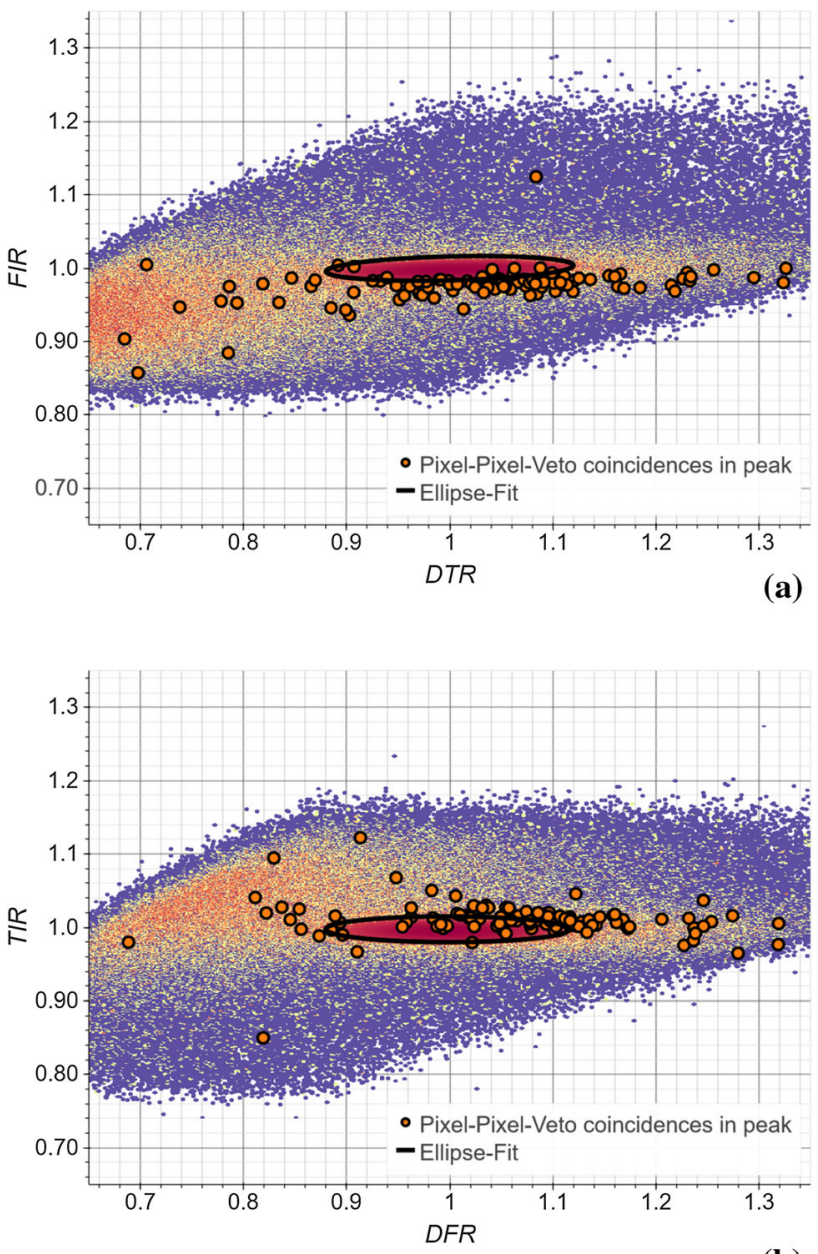

(b)

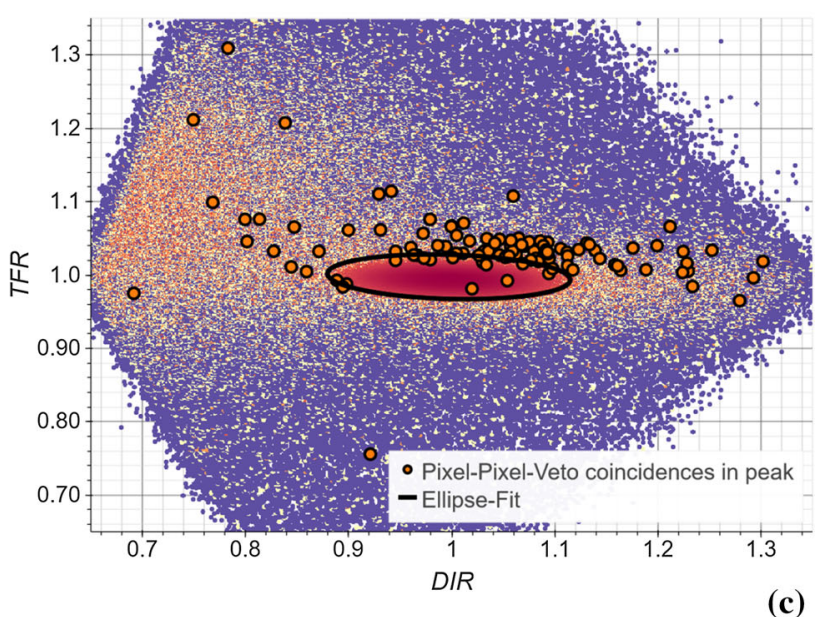

Fig. 18 The three useful parameter spaces. The ellipses are nearly not tilted and the minor/major axes have nearly similar dimensions

\section{References}

1. M. Tanabashi et al., Phys. Rev. D 98, 030001 (2018). https://doi. org/10.1103/PhysRevD.98.030001 
2. K. Altenmüller et al., Astropart. Phys. 108, 40 (2019). https://doi.org/10.1016/j.astropartphys.2019.01.003. http://www. sciencedirect.com/science/article/pii/S0927650518302597

3. K. Freund, Muonic background in the Gerde $0 \nu \beta \beta$ experiment. Ph.D. thesis, University of Tubingen (2014)

4. Y. Gornushkin, V. Vorobel, PoS ICHEP2016, 974 (2017). https:// doi.org/10.22323/1.282.0974

5. J. G. Learned, Super-Kamiokande. Underground muons in superkamiokande (1997)

6. K. Rottler, Muons in the CRESST dark matter experiment. Ph.D. thesis, University of Tubingen (2014)

7. E. Aprile et al., J. Phys. G: Nucl. Part. Phys. 40(11), 115201 (2013). https://doi.org/10.1088/0954-3899/40/11/115201

8. L. Gastaldo et al., Eur. Phys. J. Spec. Top. 226(8), 1623 (2017). https://doi.org/10.1140/epjst/e2017-70071-y

9. A. Fleischmann et al., AIP Conf. Proc. 1185(1), 571 (2009). https:// doi.org/10.1063/1.3292407

10. S. Kempf et al., J. Low Temp. Phys. 193(3), 365 (2018). https:// doi.org/10.1007/s10909-018-1891-6

11. M. Bra $\beta$, M.W. Haverkort, Abinitio calculation of the electron capture spectrum of ${ }^{163} \mathrm{Ho}$ : Auger-Meitner decay into continuum states. New J. Phys. 22(9), 093018 (2020). https://doi.org/10.1088/ 1367-2630/abac72

12. M. Braß et al., Phys. Rev. C 97, 054620 (2018). https://doi.org/10. 1103/PhysRevC.97.054620

13. S. Eliseev et al., Phys. Rev. Lett. 115, 062501 (2015). https://doi. org/10.1103/PhysRevLett.115.062501
14. L. Gamer et al., Nucl. Instrum. Methods Phys. Res. Sect. A: Accel. Spectrom. Detect. Assoc. Equip. 854, 139 (2017). https://doi. org/10.1016/j.nima.2017.02.056. https://www.sciencedirect.com/ science/article/pii/S0168900217302528

15. S. Kempf et al., J. Low Temp. Phys. 176(3), 426 (2014). https:// doi.org/10.1007/s10909-013-1041-0

16. D. Reyna, A simple parameterization of the cosmic-ray muon momentum spectra at the surface as a function of zenith angle (2006)

17. P. Shukla, S. Sankrith, Int. J. Mod. Phys. A 33(30), 1850175 (2018). https://doi.org/10.1142/S0217751X18501750

18. L. Landau, J. Phys. (USSR) 8, 201 (1944)

19. H. Bichsel, Rev. Mod. Phys. 60, 663 (1988). https://doi.org/10. 1103/RevModPhys.60.663

20. D. Wilkinson, Nucl. Instrum. Methods Phys. Res. Sect. A: Accel. Spectrom. Detect. Assoc. Equip. 383(2), 513 (1996). https://doi. org/10.1016/S0168-9002(96)00774-7. http://www.sciencedirect. com/science/article/pii/S0168900296007747

21. S. Agostinelli et al., Nucl. Instrum. Methods Phys. Res. Sect. A: Accel. Spectrom. Detect. Assoc. Equip. 506(3), 250 (2003). https://doi.org/10.1016/S0168-9002(03)01368-8. http:// www.sciencedirect.com/science/article/pii/S0168900203013688 